\title{
Lorentz Symmetry Breaking and Planar Effects from Non-Linear Electrodynamics.
}

\author{
Marcelo Botta Cantcheff ${ }^{1}$ \\ Instituto de Física Teórica (IFT/UNESP) \\ Rua Pamplona, 145 - Bela Vista \\ 01405-900 - São Paulo, SP - Brazil. \\ Centro Brasileiro de Pesquisas Fisicas (CBPF) \\ Coordenação de Física Experimental de Altas Energias (LAFEX) \\ Rua Dr. Xavier Sigaud, 150 - Urca \\ 22290-180 - Rio de Janeiro - RJ - Brazil.
}

\begin{abstract}
We propose a modification of standard linear electrodynamics in four dimensions, where effective non-trivial interactions of the electromagnetic field with itself and with matter fields induce Lorentz violating Chern-Simons terms. This yields two consequences: it provides a more realistic and general scenario for the breakdown of Lorentz symmetry in electromagnetism and it may explain the effective behavior of the electromagnetic field in certain planar phenomena (for instance, Hall effect). A number of proposals for non-linear electrodynamics is discussed along the paper. Important physical implications of the breaking of Lorentz symmetry, such as optical birefringence and the possibility of having conductance in the vacuum are commented on.
\end{abstract}

\section{Introduction.}

It is often claimed that the correct description of many electromagnetic phenomena in three dimensions is provided by the Maxwell-Chern-Simons theory [1,2]. It is believed to describe electromagnetic interactions in very thin films. However, if we accept that three-dimensionality is an effective circumstance (i.e, the world is four dimensional, but the physics of a certain phenomenon would be approximately described by a field theory on a three-dimensional manifold), a fundamental question arises: what is the mechanism that explains this behavior of the electromagnetic field from the four-dimensional point of view?

In most physical situations modelled by a planar theory, where a Chern-Simons term appears, the gauge field $A$ is considered as an effective one, describing other collective, more fundamental, degrees of freedom (fermionic or bosonic) in some material medium $[3,4]$. However, they all suppose a threedimensional manifold as its starting point $[3,4,5]$. Actually, the appearance of this term is badly motivated in planar models which effectively describe electromagnetism in thin material films. Furthermore, a dimensional reduction scheme from the ordinary Maxwell theory, interacting with charged matter in the usual way, is also hard to be argued. If we accept that at a macroscopic scale (i.e, the scale of electromagnetic interactions) the space-time is effectively four-dimensional, even in the context of electrodynamic planar phenomena, any compactification scenario is not well justified as starting point.

A few years ago, a modification of Maxwell's electromagnetism in four dimensions has been proposed which considers a kind of Chern-Simons term in the action, $\int d x^{4} V_{\alpha} \epsilon^{\alpha \beta \mu \nu} A_{\beta} F_{\mu \nu}$, where Lorentz symmetry is explicitly broken by an external vector, $V^{\mu}[6]$. There is a growing literature considering this proposal seriously, and exploring the possible origin and consequences of this approach [7].

In fact, in the present approach, we emphasize that broken Lorentz symmetry (abbreviated as BLS) would be crucial to obtain a Chern-Simons term in some three-dimensional effective model (since it is

1e-mail: botta@cbpf.br 
automatically induced from a BLS term, as will be explained in Section 1 of this paper). Reciprocally, the physical contexts for which an effective planar model with a Chern-Simons term is more appropriate [1] must dictate precisely what are the ways in which BLS should appear in a fundamental 4-dimensional electrodynamics. This is the paradigm which, together with the assumption of Lorentz invariance, motivates our construction. The model we are going to build up here assumes a non-linear (but relativistic) electrodynamics which induces a modification of this kind (BLS) of the standard Maxwell theory. Furthermore, it is shown that BLS does not need to be introduced by hand, but it can naturally appear in some realistic physical situations. For example, in the presence of external fields and/or when nonuniform distributions of charged matter are considered (and consequently, a CS term will be induced in an embedded hyperplane).

There are additional theoretical motivations for our construction. Recent works $[8,9]$ consider BLS with very interesting consequences on certain aspects of condensed matter phenomena, such as the proposal of a non-minimal coupling in order to describe the quantum Hall effect. However, the vector $V$ is identified by hand with the external magnetic field, orthogonal to the Hall sample ${ }^{2}$. In this paper we consider these observations and accomodate them as natural consequences of a more fundamental non-linear electrodynamics theory, in a general and elegant way, aiming that such ad hoc interpretations are not necessary.

This work is organized as follows: in Section 2, we define our proposal in general, writing down the basic postulates for nonlinear electrodynamics. In Section 3, we extend these assumptions to theories in which the electromagnetic field interacts with other charged ones. In Subsection 3.1, we define a nonlinear coupling to generic matter and argue on the appearance of BLS terms and non-minimal couplings. In Section 4, we discuss the Hall effect in terms of non-linear theories. In Section 5, we carry out BLS with examples of non-linear theories. We present our final observations in Section 6.

\section{Non-linear electromagnetism and BLS.}

It appears to be difficult to work with a theory whose form is unknown. However, we propose here a technique that allows (under a few simple and general assumptions) to evaluate the effective action around certain specific background solutions without the knowledge of the full non-linear theory. The assumptions are as follows:

(I) Assume that electromagnetism is described by non-linear equations. Thus, the Lagrangian $L_{N L}$ is a generic (in principle, non-quadratic), relativistically invariant, functional of a vector field $A_{\mu}$ (and $\left.F_{\mu \nu} \equiv 2 \partial_{[\mu} A_{\nu]}\right)^{3}$.

(II) There are non-trivial, stable solutions $\left(A_{\mu}^{0}, F_{\mu \nu}^{0}\right)$ of the classical equations of motion, such that small field perturbations (i.e $\left\|F_{\mu \nu}-F_{\mu \nu}^{0}\right\| \ll\left\|F_{\mu \nu}^{0}\right\|$ ) are properly described by the second order expansion of the action about these background solutions:

$$
S_{L}\left[A_{\mu}\right]=S\left[A^{0}\right]+\int d x d y\left(A_{\mu}-A_{\mu}^{0}\right)(x)\left(\left.\frac{1}{2} \frac{\delta^{2} S}{\delta A_{\mu}(x) \delta A_{\nu}(y)}\right|_{A^{0}}\right)\left(A_{\nu}-A_{\nu}^{0}\right)(y) .
$$

In particular $F_{\mu \nu}^{0} \equiv 0$ is a solution, and linearization around this one coincides with the standard Maxwell theory.

Clearly, the quantization of this theory in the saddle point approximation is contemplated in this statement because quantum fluctuations are supposed to be much weaker than the classical background fields. So, in order to quantize the electromagnetic field and find out the photon spectrum, one considers the free theory (1).

\footnotetext{
${ }^{2}$ And, in Ref. [8], BLS is used to describe the Pauli coupling [11].

${ }^{3}$ We neglect the symmetric derivatives because we only want to consider degrees of freedom corresponding to a spin- 1 boson.
} 
(III) The linearized theory around $F_{\mu \nu}^{0}=0$ has the usual $U(1)$-gauge invariance $A_{\mu} \rightarrow A_{\mu}+\partial_{\mu} \alpha$.

This assumption is motivated in the original reference [6] where gauge invariance of electrodynamics is strongly relaxed (a mass term is considered within observable limits). Here we admit this possibility, but we require that these limits depend of the strength of the background field. So, when it is weak $\left(F_{\mu \nu}^{0} \rightarrow 0\right)$, gauge invariance shall be recovered in order that the theory agrees with the full Maxwell theory in ordinary physics.

Now, we are going to show that these simple assumptions on the unknown non-linear theory are sufficient to recover the behavior that is relevant for us. The linearized effective action has the general form

$$
S_{L}\left[A_{\mu}\right]=\frac{1}{4} \int d^{4} x\left[C_{1}^{\alpha \beta \mu \nu} F_{\alpha \beta} F_{\mu \nu}+C_{2}^{\alpha \mu \nu} A_{\alpha} F_{\mu \nu}+C_{3}^{\mu \nu} A_{\mu} A_{\nu}\right],
$$

where the tensors $C_{i}$ do not depend explicitly on the spacetime point, being only algebraic functionals of the background fields, $F_{\alpha \beta}^{0}, A_{\mu}^{0}$. The crucial observation, over which our construction stands, is that the tensors $C_{i}$ are typically non-trivial, which leads to BLS.

As a first example, a very simple gauge invariant non-linear theory for pure electromagnetism is

$$
L_{\rho}\left[A_{\mu}\right]=-\frac{\beta}{4} F_{\mu \nu} F^{\mu \nu}+\frac{1}{4} \rho\left(F^{2}\right) \epsilon^{\alpha \beta \mu \nu} F_{\alpha \beta} F_{\mu \nu} .
$$

By expanding this theory about a non-constant solution, $F_{\mu \nu}^{0}(x)$, we get a BLS theory where an external vector $V_{\mu}$ appears, given by $V_{\mu}=-\frac{1}{4} \partial_{\mu} \rho\left(F_{0}^{2}(x)\right)$

Now, because we wish to discuss the consequences of this approach in some specific realistic scenario with physical interest, let us consider an example where the background solution consists of an uniform magnetic field, $B_{\mu} \equiv \epsilon_{\mu \nu \alpha \beta} t^{\nu} F^{0 \alpha \beta}$ (where $t^{\nu}=\partial^{\nu} t$ is a unit timelike four-vector and $t$ is the time coordinate in the rest frame), and the other components of $F_{\mu \nu}$ vanish. Then, clearly, $C_{i}\left[F^{0}\right]$ are algebraic functions of the vectors $B^{\mu}, t^{\nu}$, the space-time metric tensor $\eta_{\mu \nu}$, and the Levi-Civita tensor $\epsilon_{\mu \nu \alpha \beta}$. In order to simplify the analysis we assume that these tensors do not depend on $t^{\nu}$. So, by seeking all the possible independent combinations, the most general form for them reduces to:

$$
\begin{array}{r}
C_{1}^{\alpha \beta \mu \nu}=-\beta\left(B^{2}\right) h^{\alpha \mu} h^{\beta \nu} \\
C_{2}^{\alpha \mu \nu}=\rho\left(B^{2}\right) \epsilon^{\mu \nu \alpha \beta} B_{\beta} \\
C_{3}^{\mu \nu}=m\left(B^{2}\right) \eta^{\mu \nu}+k\left(B^{2}\right) B^{\mu} B^{\nu},
\end{array}
$$

where, for convenience, we have expressed $h_{\mu \nu}=\eta_{\mu \nu}-\kappa\left(B^{2}\right) B^{-2} B_{\mu} B_{\nu}$. Other possible combinations in $C_{1}$ do not contribute due to the antisymmetry of $F$, and many other ones (for instance $\epsilon^{\mu \nu \alpha \beta} A_{\alpha} \partial_{\beta} F_{\mu \nu}$ ), were dropped out because they may be absorbed into $C_{2}$ and/or $C_{3}$, or contribute to boundary terms (e.g $\epsilon^{\mu \nu \alpha \beta} F_{\mu \nu} F_{\alpha \beta}$ ). There also appear terms depending on $\partial_{\mu} A^{\mu}$ or $k_{\mu} A^{\mu}$ (for some vector $k_{\mu}$ ) but they are not gauge invariant and could be considered to be part of the gauge-fixing sector of the theory.

Gauge invariance of $S_{L}$ implies additional restrictions on these general combinations: $C_{3}$ must be antisymmetric, and therefore $C_{3}=m=k=0$. Furthermore $\partial_{\mu} C_{2}^{\alpha \mu \nu}=0$ implies $\partial_{[\mu}(\rho B)_{\nu]}=0$ which is satisfied for a constant $B_{\mu}$. Finally, by virtue of assumption (II), the coefficients must satisfy $\rho(0) \in \Re, h(0)=0$.

So, the resulting gauge invariant part of the Lagrangian is precisely the Maxwell-CS theory in four dimensions

$$
L_{L}\left[A_{\mu}\right]=-\frac{\beta}{4} F_{\mu \nu} F^{\mu \nu}+\frac{1}{4}\left(\rho B_{\alpha}\right) \epsilon^{\alpha \beta \mu \nu} A_{\beta} F_{\mu \nu}
$$

proposed by Carroll, Field and Jackiw [6]. This theory breaks Lorentz symmetry for the external background vector $V_{\mu}=\rho B_{\mu}$, which shows that the identification speculated in [9] could be derived in a framework of this type.

Let us briefly explain why the Chern-Simons action, automatically appears in the BLS action when we search for planar features (thus turning dimensional reduction unnecessary, as commented in the 
introduction). In fact the BLS action is actually a CS theory in $(2+1)$-dimensions embedded in $(3+1)$ dimensions, and by itself, it does not encode any information on the field-dependence in the direction of the external (for instance, space-like) vector $V$ : if $z$ is its affine parameter, i.e. $V=\frac{\partial}{\partial z}$, then we get a foliation of the spacetime in $(2+1)$-hypersurfaces $\Sigma_{z}$ parametrized by $z$ (and $V$ is orthogonal to each hypersurface ${ }^{4}$ ). Therefore, the BLS action may be written as

$$
S_{B L S}=\int_{0}^{L} d z S_{C S}\left[A(z), \Sigma_{z}\right]
$$

where

$$
S_{C S}\left[A(z), \Sigma_{z}\right]=\int_{\Sigma_{z}} A(z) \wedge d A(z),
$$

is the Chern-Simons action for the 1-form gauge field $A(z)$ on a three-dimensional manifold $\Sigma_{z}$. Thus, the dependence of this field on the parameter $z$ is not determined by this theory. It only has to satisfy usual convergence conditions. For example, if the interval $(0, L)$ extends to $(-\infty,+\infty), A(z)$ has to be an square-integrable function $\left(A \in L^{2}(\Re)\right)$. In this sense, we can interpret the BLS action simply as a sum of Chern-Simons theories on manifolds $\Sigma_{z}$.

Consequently, at the low-energy limit of the electromagnetic field, the Maxwell term in (5) is negligible for the planar dynamics and then we get a planar Chern-Simons theory without any dimensional reduction (which would have to be further justified ${ }^{5}$ ). Notice that this describes an eventual situation of confinement of the electromagnetic field (photon) into a $(2+1)$-manifold, which does not result from a constraint of the charged matter into a planar sample.

Let us point out that away from the low energy regime (i.e, when the Maxwell term contributes), there could also be a totally planar phase, where the contribution of the term $V^{\mu} F_{\mu \nu}$ to the action would become negligible. Since the coefficients $\beta, \theta, h, .$. depend on the particular non-linear theory, the condition for this is that $h_{\mu \nu}$ is the flat 2+1-metric $h_{\mu \nu}=\eta_{\mu \nu}-B^{-2} B_{\mu} B_{\nu}$ for some value of the background field. For instance, the function $\kappa\left(B^{2}\right)$ could go to 1 as $B^{2} \rightarrow \infty$. In this case we would not only have BLS, but exact planar Maxwell-Chern-Simons electromagnetism in the presence of a strong background field $B_{\mu}$. In Section 4 we argue on a planar projection, in the context of the Hall effect.

It is interesting to notice that this BLS-term which, as shown here, is nothing but a genuine ChernSimons term (in $(2+1)$ dimensions). It may also be obtained (see reference [10]) by considering the effective action of a standard (Lorentz and CPT invariant) gauge theory in four dimensional Minkowski space-time, such that a spatial dimension is taken to be compact $\left(\sim M \times S^{1}\right)$. Then, by integrating out the chiral fermions while keeping gauge invariance, a Chern-Simons theory on the uncompactified part (three-dimensional) of spacetime $M$ appears.

This is remarkable because, despite what was said in the last paragraph, we are not considering nontrivial space-time topologies. This opens the possibility of obtaining such a term, alternatively, in a more standard electrodynamics theory in which the background would have non-trivial topology. However, as it happens in all approaches based on compactification of the space-time, it is not clear what is the physical reason behind this compactification.

\section{Non-linear electrodynamics: BLS in matter backgrounds.}

If we consider non-linear electrodynamics involving possible non-linear couplings between charged fields and the electromagnetic potential, BLS could also appear due to special (background) distributions of charged matter (thin distributions, for instance).

So, the possibility we are going to present here shall provide a more realistic phenomenological context in which BLS could appear. Up to now, BLS was most related to astrophysical situations $[6,12,13]$ or

\footnotetext{
${ }^{4}$ Notice that if the space-time (or the space-time region considered in the integration) is simply connected, the condition of existence of this $z$-coordinate is equivalent to gauge invariance of the action, namely $d V=0$.

${ }^{5}$ Alternatively, a dimensional reduction scheme may be considered [8] when $V$ does not coincide with the coordinate of reduction, but in this case, the part of the action which depends on derivatives in the $V$-direction is lost.
} 
very high energy physics [14], rather than to the presence of matter or background fields in general. In the present approach, we wish to introduce this point of view.

Thus, one must assume a total (non-linear) action for electrodynamics

$$
S_{E D}=S\left[A, F, \bar{\psi}, \psi, \phi, \phi_{\mu}\right],
$$

where $\bar{\psi}, \psi$ are fermionic charged fields and $\phi, \phi_{\mu}$ describe bosonic matter fields with spin- 0 and -1 , respectively. We will not make here any hypothesis on the nature of these fields. However, one experimentally knows that electromagnetism in the vacuum is accurately described by the observed Maxwell theory. Thus, the only possibility of having some deviation at some observable level is, perhaps, near or in the interior of certain special distributions of matter (e.g. thin films) ${ }^{6}$. Therefore, these fields could be interpreted as effective ones, describing perhaps, collective degrees of freedom associated to the material medium. So, for example, these fields could be describing phonons, which are believed to be coupled to the electromagnetic field in material media, or some condensate of fundamental charged fields (this is the case of the Cooper-pairs, which should be important to describe the superconductivity phenomena).

So, from now on, we assume that the postulates (I, II, III) extend to theories in which the electromagnetic field interacts with other ones.

Next, let us assume, for simplicity, a background distribution of a charged scalar field, $\phi_{0}(x)$. Following the procedure established in Section 1, arguing the validity of equation (1), we also get tensors $C_{i}=$ $C_{i}\left(\phi_{0}, \partial_{\mu} \phi_{0}, \partial_{\mu} \partial_{\nu} \phi_{0}, \ldots\right)$ as in expression (4). We assumes, for simplicity, that the degrees of freedom involved in the electromagnetic interaction are precisely the ones corresponding to a standard charged spin-0 boson. Also, to avoid the appearance of ghosts, second (or higher) order derivatives will not be considered in the action. So, we get $C_{i}=C_{i}\left(\phi_{0}, \partial_{\mu} \phi_{0}\right)$. In particular, the contribution to BLS is given by $C_{2}$ (or equivalently $C_{1}$ ), which in general reads:

$$
C_{2}^{\alpha \mu \nu}=\epsilon^{\mu \nu \alpha \beta} \partial_{\beta} \rho\left(\phi_{0}\right)=\rho^{\prime}\left(\phi_{0}\right) \epsilon^{\mu \nu \alpha \beta} \partial_{\beta} \phi_{0} \quad\left(\Rightarrow V_{\mu}=\nabla_{\mu} \rho\right) .
$$

Thus, the most general linearized gauge invariant Lagrangian for electromagnetism in a background $\phi_{0}$ is:

$$
L_{e f f}\left[A_{\mu}\right]=-\frac{\beta\left(\phi_{0}\right)}{4} F_{\mu \nu} F^{\mu \nu}+\frac{\rho\left(\phi_{0}\right)}{4} \epsilon^{\alpha \beta \mu \nu} F_{\alpha \beta} F_{\mu \nu} .
$$

Clearly, for nearly uniform distributions ( $\phi_{0} \approx c t e$ ), the second term turns out to be a boundary term, and we recover the standard Maxwell theory without BLS as expected.

It is not difficult to build up a toy model for electromagnetic field interacting with bosonic spin-0 matter field such that this theory is obtained by linearization. For example, one could keep a non-selfinteracting electromagnetic field and encode the non-linearity in the interaction term as in $L_{E D}\left[A_{\mu}, \phi\right]=$ $-\frac{1}{4} F_{\mu \nu} F^{\mu \nu}+\frac{\rho(\phi)}{4} \epsilon^{\alpha \beta \mu \nu} F_{\alpha \beta} F_{\mu \nu}+L_{\phi}\left(\partial_{\alpha} \phi, \phi\right)$. Further expanding around a solution $\phi_{0}(x)$ (and $F_{\alpha \beta}^{0}$ ) of the equations of motion ${ }^{7}$ one recovers the free theory (10).

This approach should be helpful to model electrodynamical phenomena (e.g. superconductivity) in material media, where non-homogeneities, as some preferred structure of planes described by the gradient $\nabla \rho$, are supposed to be important. In particular, notice that a BLS term would appear in the interface between two different material media if we consider Goldstone/Higgs-like Lagrangians $L_{\phi}$, and different vacuum expectation values in each medium (domain). This proposal will be explored in more detail in a forthcoming work.

Next, we present another example where electromagnetism is coupled to vectorial bosonic matter, and exhibits spontaneous breaking of Lorentz symmetry ${ }^{8}$. This is a particular case of non-linear electrodynamics where the background vector $V_{\mu}$ corresponds to the vacuum configuration of the field $\phi_{\mu}$.

\footnotetext{
${ }^{6} \mathrm{Or}$ in presence of external electromagnetic field in a non-linear theory, as argued in the first section.

${ }^{7}$ It may be even a vacuum solution.

${ }^{8} \mathrm{~A}$ close example was given in Ref. [15].
} 


$$
L\left[A_{\mu}, \phi_{\mu}\right]=-\frac{\beta}{4} F_{\mu \nu} F^{\mu \nu}+\frac{1}{4} \phi_{\alpha} A_{\beta} \epsilon^{\alpha \beta \mu \nu} F_{\mu \nu}+\frac{\lambda}{4} G_{\mu \nu} G^{\mu \nu}+V\left(\phi_{\mu} \phi^{\mu}\right),
$$

where $V$ is a non-linear function and

$$
G_{\mu \nu} \equiv \partial_{\mu} \phi_{\nu}-\partial_{\nu} \phi_{\mu}
$$

This is not $U(1)$-invariant but it is Lorentz invariant. When this symmetry is spontaneously broken, gauge invariance is restored in the action for the free fields.

In the vacuum state for this field, the kinetic term vanishes $G^{2}=0$, and the maximally symmetric solution corresponds to $G_{\mu \nu}=0$. Similarly we have $F_{\mu \nu}=0$. A local solution of this is

$$
\phi_{0 \mu}=\partial_{\mu} \phi_{0}, A_{0 \mu}=0 .
$$

If $\left.V^{\prime \prime}\right|_{0}<0$, we have a minimum and $\phi_{0 \mu}(x)=\partial_{\mu} \phi_{0}$ must be obtained as a non-trivial solution of the non-linear differential equation $V^{\prime}\left(\partial_{\mu} \phi_{0} \partial^{\mu} \phi_{0}\right)=0$. Substituting these vacuum state configurations in expression (1) we obtain the non-Lorentz invariant (but gauge invariant ${ }^{9}$ ) theory:

$$
L\left[A_{\mu}, \phi_{\mu}\right]=-\frac{\beta}{4} F_{\mu \nu} F^{\mu \nu}+\frac{1}{4}\left(\partial_{\alpha} \phi_{0}\right) A_{\beta} \epsilon^{\alpha \beta \mu \nu} F_{\mu \nu}+\frac{\lambda}{4} G_{\mu \nu} G^{\mu \nu}+\left.\frac{1}{2} V^{\prime \prime}\right|_{0}\left(\phi_{\mu}-\partial_{\mu} \phi_{0}\right)^{2} .
$$

\subsection{General action for matter and non-linear coupling to electromagnetism.}

We consider here a general action for the matter fields with the standard minimal coupling to the electromagnetic field, but with an additional non-linear coupling term. We shall argue that when these sources satisfy an obvious physical requirement (existence of rest charges), BLS is generated in our approach. The proposed non-linear coupling is specially important for our purposes, and it presents another important property related with a non-minimal coupling, recently worked out with very interesting physical consequences [8].

Let us propose the following action for fundamental electrodynamics:

$$
S=\int d x^{4}\left(-\frac{\beta}{4} F_{\mu \nu} F^{\mu \nu}+k\left(\epsilon_{\alpha \rho \sigma \tau} j^{\rho} F^{\sigma \tau}\right)\left(\epsilon^{\alpha \beta \mu \nu} A_{\beta} F_{\mu \nu}\right)+A_{\mu} j^{\mu}\right)+S_{\text {source }}[\bar{\psi}, \psi, \phi, \ldots] .
$$

Remarkably, this theory satisfies assumptions I, II and III. In particular, if the minimally coupled theory $\int d x^{4} A_{\mu} j^{\mu}+S_{\text {source }}$ is gauge invariant, then (15) will satisfy III.

As anticipated, we do not give here any explicit free dynamics for the sources (which determine $j^{\mu}$ ), but we enunciate a very natural physical condition which $j^{\mu}$ must satisfy as an additional assumption:

(IV) (Existence of rest charge): the action $S_{\text {source }}$ is such that the equations of motion derived from (15) admit a background solution where the source is a charge at rest. In other words, there exists a classical solution where the current is

$$
j^{\mu}=q t^{\mu},
$$

where $q$ is a function of the spatial coordinates.

When (16) is imposed, a solution $F_{0}$ of the equations of motion exists. So, we get $\epsilon_{\alpha \rho \sigma \tau} j^{\rho} F_{0}^{\sigma \tau}=q B_{\alpha}$, where $B_{\alpha}$ is, by definition, the magnetic field in the rest frame. Thus, it is clear that when we consider expansion (1), there appears a BLS contribution:

$$
S_{B L S}=\int d^{4} x\left(q k B_{\alpha}\right) \epsilon^{\alpha \beta \mu \nu} A_{\beta} F_{\mu \nu}
$$

where the external vector is proportional to the background magnetic field, as argued in Section 1 (eq. (5)). As we can see, this is specially relevant to the study of the Hall effect (next Section).

\footnotetext{
${ }^{9}$ The $U(1)$ gauge invariance is: $\phi_{\mu} \rightarrow \phi_{\mu} \quad, \phi \rightarrow \phi \quad, A_{\mu} \rightarrow A_{\mu}+\partial_{\mu} \alpha$.
} 
Notice, in addition, that another term appears from expansion (1) which remarkably coincides with the non-minimal coupling introduced in [8]:

$$
S_{N M}=\int d^{4} x V_{\alpha} \epsilon^{\alpha \beta \mu \nu}(j+J)_{\beta} F_{\mu \nu},
$$

where $J$ is a current describing a perturbation of the source (16). The BLS vector is $V^{\alpha}=\epsilon^{\alpha \beta \mu \nu} A_{0 \beta} F_{0 \mu \nu}$. As we will see in Section 4 (eq. 30), if the non-linear theory is not gauge invariant (but satisfies III), $V_{\mu}$ may coincide with the direction of $B_{\mu}$ even for a background solution $F_{0}=$ constant as speculated in [9].

As we mentioned above, these non-minimal couplings have been exhaustively studied also in the context of BLS, but with a $V$ that has not been generated in the context of the model [8]. Moreover, this opens the possibility to explain the non-minimal Pauli-like coupling [11].

Let us briefly mention that there is another similar theory with a non-linear coupling, which is gauge invariant from the beginning (if $S_{\text {source }}$ is also):

$$
S=\int d x^{4}\left(-\frac{\beta}{4} F_{\mu \nu} F^{\mu \nu}+k\left(\epsilon_{\alpha \rho \sigma \tau} j^{\rho} F^{\sigma \tau}\right)\left(\epsilon^{\alpha \beta \mu \nu} j_{\beta} F_{\mu \nu}\right)+A_{\mu} j^{\mu}\right)+S_{\text {source }}[\bar{\psi}, \psi, \phi, \ldots] .
$$

By repeating the above procedure (to expand around solutions (16)), there appear terms in the lagrangian corresponding precisely to non-minimal or Pauli coupling (proportional to $B_{\alpha} \epsilon^{\alpha \beta \mu \nu} J_{\beta} F_{\mu \nu}$ ). This presents the main properties of the model proposed in ref. [9] in $2+1$ dimensions (in the next Section, it will become more clear how the planar confinement may appear in a four-dimensional theory of this type).

\section{Planar electromagnetism and Hall effect}

Quantum Hall effect is the phenomenon of a longitudinal electric field yielding a transverse current in the presence of an external perpendicular magnetic field $B_{\mu}$. The effective physics of this phenomenon is described by an effective Maxwell-Chern-Simons (or pure Chern-Simons) theory on a plane perpendicular to $B_{\mu}[3,4,5]$. However, as discussed in the introduction of the paper, such description is not clearly well argued from a four-dimensional viewpoint.

In these models, the vector field is the so-called "statistical field" which encodes the effective dynamics of many charged bodies in the Hall sample and part of the pure electromagnetic field $A_{\mu}$. Furthermore, an additional external electromagnetic field $A_{\mu}^{0}$ is considered in order to describe the field $B_{\mu}[4]$.

In the present framework, we are able to reproduce most of this behavior even at the classical level without making any reference to the microscopic structure/dynamics of the charged matter in the conductor, but based on a possible non-linearity intrinsic to electrodynamics.

In our approach, the presence of an external magnetic field automatically implies the appearance of a BLS term (eq. (5)), and no kind of dimensional reduction is necessary to obtain a pure Chern-Simons term in the plane of conduction. It may be obtained from (5) and, consequently, from a non-linear theory of electromagnetism, by an elegant and very simple procedure.

Notice that Ohm's law, $J^{i}=\sigma^{i j} E_{j}$ is usually presented as an effective (constitutive) relation arising from the collective processes that occur in a conducting medium. They are used to allow the Maxwell equations to describe the propagation of electromagnetic fields in material media and they do not follow from fundamental electrodynamics. Remarkably, in the class of theories that we are proposing here, this relation may be obtained from the equations of motion and it does not need be imposed by hand.

Let us assume the existence of an uniform background magnetic field $B^{\nu}$ in a Hall sample placed in a region $R_{H}$ of the spacetime. Consider also a non-linear theory such that, by the procedure described above, the electromagnetic sector minimally coupled with an external source reads:

$$
S=\int_{R_{H}} d x^{4}\left(L_{E M}+A_{\mu} J^{\mu}\right)
$$

where $L_{E M}$ is given by (5) (in the previous Section we proposed a realization of this (see eq. (17) where the coefficient $\rho$ may be identified with $k q$ ) ) and $J^{\mu}$ is the electric current that should be produced in the 
Hall sample. This description in which $B_{\mu}$ is treated as a fixed background field is actually appropriate, since in the QHE, typically $|B| \sim 1 \mathrm{~T}$.

Then, the equation of motion derived from this action is:

$$
\frac{\beta}{2} \partial^{\nu} F_{\nu \mu}=\frac{1}{2} \rho\left(B^{2}\right) B^{\nu} \epsilon_{\mu \nu \alpha \beta} F^{\alpha \beta}-J_{\mu} ;
$$

thus, for a nearly constant electromagnetic field $F$ (or for low energy), the LHS of this equation is negligible and we can write

$$
\frac{1}{2} \rho\left(B^{2}\right) B^{\nu} \epsilon_{\mu \nu \alpha \beta} F^{\alpha \beta}=J_{\mu} .
$$

By contracting this equation with $B^{\mu}$, one gets

$$
J_{\mu} B^{\mu}=0,
$$

which is the condition for planar confinement, expected to be valid in the context of the Hall effect.

Considering the spatial components of this equation, we obtain that the current flux will be deviated by the electric field according to the equation:

$$
\frac{1}{2} \rho|B| \epsilon_{i j} E^{j}=J_{i} .
$$

So, the Hall conductance can be expressed in terms of parameters of the non-linear theory and as a function of $B_{\mu}$ :

$$
\sigma_{H}=\frac{1}{2}|B| \rho,
$$

and the rest charge density is

$$
J_{0}=\frac{1}{2} \rho B^{\mu} b_{\mu},
$$

where $b_{\mu} \equiv \epsilon_{\mu \nu \alpha \beta} t^{\nu} F^{\alpha \beta}$ is the fluctuation of the magnetic field (i.e, the total magnetic field is $B_{\mu}+b_{\mu}$ ).

Finally, according to assumption II, viewed as a saddle point approximation, one finds that, to low energies, the Chern-Simons theory (22) effectively describes the Hall effect, and $\sigma_{H}$ results to be quantized in fractions of $e^{2} / h$ in the usual way (see the standard arguments in refs. [3, 4, 16]). Actually, we can give here a simple argument for it: let us assume that the magnetic flux ${ }^{10}$ through the total sample area ${ }^{11}$, $\Phi=N_{\Phi}=\int_{S_{H}} \vec{b} \cdot d \vec{a}$ is an integer (in units in which $e=\hbar=1[4]$ ). On the other hand, the number of charges must be $N e=\int_{S_{H}} J^{0} d a$. Thus, substituting these two expressions into (25), and using that $\vec{B} . \vec{b} d a=|B| \vec{b} . d \vec{a}$, we get

$$
N=\left(\frac{1}{2} \rho|B|\right) N_{\phi}=\sigma_{H} N_{\Phi} \Rightarrow \sigma_{H}=\frac{N}{N_{\Phi}} .
$$

So, if each electron is attached to an integer number of flux units [17], $\sigma_{H}$ is the inverse of this number. In a forthcoming work we shall analyze this result by considering quantum aspects of the electric charges and the conductor. The focus here has been on the description of the behavior of the electromagnetic field in this situation.

The above discussion is especially meaningful for the non-linear models which involve matter fields, as presented in the previous Section (where $\rho$ is given by the background matter fields), with Ohm's law appearing naturally as expected and having a natural interpretation. However, let us point out that an interesting question arises here: "is it possible a vacuum contribution to the Ohm conductivity law out of material media?". If pure electromagnetism with $\rho\left(B^{2}\right) \neq 0$ exists $^{12}$, then, there exists an intrinsic

\footnotetext{
${ }^{10}$ Due to the dynamic part of the magnetic field $b$.

${ }^{11}$ As we explained in the end of Section 2 , one can take the region $R_{H}$ to be $S_{H} \times(0, L) \times \Re$ where $S_{H}$ is the sample plane (two-dimensional), and $L$ may be arbitrarily small.

${ }^{12}$ In other words, if there is a classical solution to the non-linear theory such that the matter fields vanish and $B_{\mu}(x)=$ constant $\neq 0$ in some region $R_{H}$.
} 
contribution of the electromagnetic field in itself to Ohm's law and consequently, a vacuum contribution (due to the electromagnetic background) to the conductance ${ }^{13}$.

The conventional approach to the quantum Hall effect (QHE) says that it only occurs in conducting materials. The vector potential is considered as the sum of an effective field, representing the collective degrees of freedom of many interacting charged bodies in the conductor, plus the pure electromagnetic vector potential. In our approach, these degrees of freedom are encoded in the other fields (here referred to as "matter fields") but furthermore a material medium would not be required to obtain this effect, if pure non-linear theories exist. In the next section we analyze an example of this situation.

\section{Non-linear toy models.}

In this section, we discuss some examples of non-linear electrodynamics which exhibit BLS.

First, let us analyze the case of pure electromagnetism, where BLS can occur without matter fields, due to the presence of an intense magnetic field. The following example is the most interesting for us since it satisfies all the assumptions I - IV and is similar to the example discussed in a generic way in Section 1. We consider the non-linear theory for pure electromagnetism as being given by:

$$
\mathcal{S}_{N L}\left[A_{\mu}\right]=\int d x^{4}\left(\frac{\beta}{4} F_{\mu \nu} F^{\mu \nu}+\frac{\mu}{4} W_{\mu \nu \alpha} W^{\mu \nu \alpha}\right),
$$

where

$$
W=F_{[\mu \nu} A_{\alpha]} .
$$

Let us consider the following ansatz for a classical solution

$$
A_{0 \mu} \equiv \epsilon_{\mu \nu \alpha \beta} x^{\nu} B^{\alpha} t^{\beta}+c t_{\mu},
$$

where $B_{\mu}$ and $c$ are constant. We can directly verify that

$$
{ }^{\star} W_{0 \mu} \equiv{ }^{\star} W_{\mu}\left[A_{0}\right]=-c B_{\mu},
$$

where ${ }^{\star} W_{\beta} \equiv \epsilon_{\beta}{ }^{\mu \nu \alpha} F_{[\mu \nu} A_{\alpha]}$. To verify that this is actually a solution of the theory (27), we observe that the equations of motion derived from this action are:

$$
\partial^{\mu} F_{\mu \nu} \propto A^{\alpha} \partial^{\mu} W_{\mu \nu \alpha} .
$$

Then, (29) is a trivial solution since $F_{0}, W_{0}$ are constant. Linearization of this theory around this solution gives gauge-dependent terms, Maxwell-like terms (as in the model (5)), and a BLS contribution given by

$$
\mathcal{S}_{B L S}=\int d x^{4} \frac{\mu}{2}{ }^{\star} W_{0 \beta} \epsilon^{\beta \mu \nu \alpha} W_{\mu \nu \alpha}=-\int d x^{4} \frac{\mu}{2} c B_{\beta} \epsilon^{\beta \mu \nu \alpha} F_{\mu \nu} A_{\alpha},
$$

as we expected.

Notice that the theory defined by (27) is not gauge invariant. Assumption III asserts that linearized electromagnetism about $F=0$ must be gauge invariant, but this is not necessarily true for the full non-linear theory. However, we can construct a non-linear gauge invariant theory with a similar solution, assuming a non-linear coupling with matter fields.

A similar non-linear theory of electromagnetism coupled to a scalar field is

$$
\mathcal{S}_{N L}\left[\bar{\phi}, \phi, A_{\mu}\right]=\int d x^{4}\left(\frac{1}{2} D^{\mu} \bar{\phi} D_{\mu} \phi+\frac{\beta\left(|\phi|^{2}\right)}{4} F_{\mu \nu} F^{\mu \nu}+\frac{\mu}{4 e^{2}} \bar{\rho}_{\mu \nu \alpha} \rho^{\mu \nu \alpha}\right)
$$

where

$$
\rho_{\mu \nu \alpha}=F_{[\mu \nu} D_{\alpha]} \phi, \quad \bar{\rho}_{\mu \nu \alpha}=F_{[\mu \nu} D_{\alpha]} \bar{\phi}
$$

\footnotetext{
${ }^{13}$ Given a background magnetic field $B_{\mu}$, then, in these theories, the external sources $J_{\mu}$ and the electromagnetic field $F_{\mu \nu}$ will be related by (23) and (25)
} 
and

$$
D_{\mu} \phi=\left[\partial_{\mu}-i e A_{\mu}\right] \phi, D_{\mu} \bar{\phi}=\left[\partial_{\mu}+i e A_{\mu}\right] \bar{\phi} .
$$

This theory is invariant under local $U(1)$ gauge transformations:

$$
\phi^{\prime}=e^{i \alpha} \phi, \quad \bar{\phi}^{\prime}=e^{-i \alpha} \bar{\phi}, \quad A_{\mu}=A_{\mu}-i e \partial_{\mu} \alpha
$$

As a particular case of (33), we could also consider a non-standard scalar field whose dynamics is given in the non-minimal interaction with the electromagnetic field, namely the $\mathrm{U}(1)$-invariant theory:

$$
\mathcal{S}_{N L}\left[\bar{\phi}, \phi, A_{\mu}\right]=\int d x^{4}\left(\frac{|\phi|^{2}}{4} F_{\mu \nu} F^{\mu \nu}+\frac{\mu}{4 e^{2}} \bar{\rho}_{\mu \nu \alpha} \rho^{\mu \nu \alpha}\right) .
$$

Notice that, due to (35), there clearly appears a term $\mu \bar{\phi} \phi W_{\mu \nu \alpha} W^{\mu \nu \alpha}$ in the lagrangian, like in (27). A solution similar to (29) may be found in this theory but, in this case, the second term can be eliminated by a gauge transformation and carried to the $\phi$ sector. It is straightforward to verify that a background configuration which yields a BLS term (32) is:

$$
\begin{array}{r}
\phi(t)=\zeta e^{i c e t}, \quad \zeta=\text { constant }, \quad c^{2} \mu=1, \\
A_{0 \mu} \equiv \epsilon_{\mu \nu \alpha \beta} x^{\nu} B^{\alpha} t^{\beta} .
\end{array}
$$

This corresponds to a very special $\phi$ which could be interpreted as an auxiliary field in order to restore gauge invariance rather than describing any sort of physical matter.

\section{Remarks and Outline.}

There are many reasons to believe that a fundamental theory should be relativistic. We have seen that it is possible to get BLS in the electromagnetic theory from some relativistic non-linear theory and to reproduce, consequently, many features of planar electrodynamics. If electrodynamics is indeed nonlinear, many new physical effects should be expected. In particular, optical activity (birefringence) is a common feature of electromagnetism with BLS [13], which is a general characteristic of the theories we are considering. Finally, let us remark that the mechanisms proposed here to obtain BLS emphasizes more the possibility of having BLS in material media, where strong background electromagnetic fields or special charge distributions may occur or may be prepared in experiments.

This kind of analysis might be performed in more general contexts, like non-Abelian gauge theories. In particular, it would be interesting to pursue a similar approach in gauge formulations of gravity. Our point of view would be in sharp contrast to the standard one on this subject, where BLS appears at very large scales, related to cosmic anisotropies [6,12,13], or in the context of superstring physics [14]. Such anisotropies of matter at large scale should give place to BLS due to a non-linear effective electrodynamics in the way we have proposed here.

\section{Acknowledgements}

The author thanks A.L.M.A. Nogueira and Ricardo Paschoal for fruitful discussions. S. Alves Días and José A. Helayël Neto are especially acknowledged for suggestions and criticisms on the manuscript. CLAF and $\mathrm{CNPq}$ are acknowledged for the invaluable financial help.

\section{References}

[1] The most important original paper is: S. Deser, R. Jackiw and S. Templeton, Ann. Phys. 140 (1982) 372-411, [Erratum-ibid. 185 (1988) 406], reprinted in ibid. 281 (2000) 409-449. More recent and introductory articles are: G.V. Dunne, "Aspects of Chern-Simons Theory," http://arXiv.org/abs/hepth/9902115; R. Jackiw, "Topics In Planar Physics," MIT-CTP-1824 DIAS Working Seminar, Dublin, 
Ireland, May 1989: 19th GIFT School, Jaca, Spain, Jun 1989: 8th Symp., Mt. Sorak, Korea, Jul 1989: Banff Summer School, Aug 1989: 29th Schladming School, Schladming, Austria, Mar 1990.(refs de Chern simons en 3 dim eletrodinamica)

[2] W.A. Moura-Melo and J.A. Helayël-Neto, electrodynamics in D = (2+1)-dimensions," Phys. Rev. D 63 (2001) 065013, electrodynamics in D = (2+1)," hep-th/0004143; W.A. Moura-Melo, Peculiar Aspects of Planar Maxwell and Maxwell-Chern-Simons Electrodynamics, Ph.D. Thesis, CBPF, 2001 (avaiable at http://www.cbpf.br/ dcp/papers/).

[3] S.C. Zhang, T.H. Hansson, S. Kivelson, Phys. Rev. Lett. 62 (1988) 82.

[4] A. Lopez and E. Fradkin, Phys. Rev. B 44 (1991) 5246.

[5] R.E. Prange and S.M. Girvin (Eds.), The Quantum Hall Effect, 2nd. ed., Springer-Verlag, New York, 1990; S. Das Sarma and A. Pinczuk (Eds.), Perspectives in Quantum Hall Effects, Wiley, New York, 1997; O. Heinonen (Ed.), Composite Fermions, World Scientific, Singapore, 1998. (CS y FQHE)

[6] S. M. Carroll, G. B. Field, Roman Jackiw, Phys.Rev.D41:1231,1990

[7] C. Adam, F.R. Klinkhamer, Nucl.Phys. B607 (2001) 247-267 and Phys.Lett. B513 (2001) 245-250 ; A. Kostelecky, R. Lehnert, Phys.Rev. D63 (2001) 065008 ; A. A. Andrianov, R. Soldati, L. Sorbo, Phys.Rev. D59 (1999) 025002; R. Jackiw, A. Kostelecky, Phys.Rev.Lett. 82 (1999) 3572-3575; J.-M. Chung, B. K. Chung, Phys.Rev. D63 (2001) 105015; J.-M. Chung , Phys.Rev. D60 (1999) 127901; M. Perez-Victoria, Phys.Rev.Lett. 83 (1999) 2518-2521; G. Bonneau, Nucl.Phys. B593 (2001) 398-412; M. Perez-Victoria, JHEP 0104 (2001) 032.

[8] H. Belich, M.M. Ferreira Jr., J.A. Helayl-Neto, M.T.D. Orlando Phys.Rev. D67 (2003) 125011 (hepth/0212330); H. Belich, M.M. Ferreira Jr., J.A. Helayl-Neto, M.T.D. Orlando Phys.Rev. D68 (2003) 025005 (hep-th/0301224 ); H. Belich, M.M. Ferreira Jr., J.A. Helayel-Neto Nucl.Phys.Proc.Suppl. 127 (2004) 105 (hep-th/0305089) M.M. Ferreira, Phys.Rev.D70 (2004) 045013.

[9] R. C. Paschoal, J. A. Helayel-Neto, L. P.G. de Assis, CBPF-NF-018-04, hep-th/0407109; and R. C. Paschoal, J. A. Helayel-Neto, Phys. Lett. A 313 (2003) 412.

[10] F.R. Klinkhamer, NPB 578 (2000) 277, hep-th/9912169.

[11] J. Stern, anyons, Phys. Lett. B 265 (1991) 119-123. I.I. Kogan, Phys. Lett. B 262 (1991) 83-87. S.M. Latinsky and D.P. Sorokin, JETP Lett. 53 (1991) 187-189; Mod. Phys. Lett. A 6 (1991) 3525-3530, and references therein. H. Belich, T. Costa-Soares, M.M. Ferreira Jr., J.A. Helayel-Neto, M.T.D. Orlando Gauge Theories with Lorentz-symmetry Violation and Electrically Charged Vortices in the Planar Regime (hep-th/0407260); S.Ghosh, Pauli Term, Anyons, "Cooper Pair", ... or Noncommutative Maxwell-Chern-Simons, hep-th/0407086.

[12] S. M. Carroll, George B. Field, Phys. Rev. Lett. 79 (1997) 2394-2397 Is there evidence for cosmic anisotropy in the polarization of distant radio sources?; J. W. Moffat, Int. J. Mod. Phys. D12 (2003) 1279-1288; E. O. Iltan, Mod. Phys. Lett. A19 (2004) 327-336. S. Coleman, S. L. Glashow, Phys.Rev. D59 (1999) 116008

[13] M. Goldhaber, V. Trimble, J. Astrophys. Astron. 17:17,1996; V.B. Bezerra, H.J. Mosquera Cuesta, C.N. Ferreira, Phys.Rev. D67 (2003) 084011

[14] V. Alan Kostelecky, S. Samuel, Spontaneous Breaking Of Lorentz Symmetry In String Theory, Phys. Rev. D39 (1989) 683

[15] L.P. Colatto, A.L.A. Penna, W.C. Santos; Eur.Phys.J. C36 (2004) 79-87 (hep-th/0310220).

[16] R. B. Loughlin, Phys. Rev. Lett. 50 (1983) 1395.

[17] J. Jain, Phys. Rev. Lett. 63 (1989) 199. 